\section{WIRELESS TECHNOLOGY}

\section{Beaming with energy}

J. Appl. Phys. 121, 014901 (2017)

The ways in which we use wireless devices seem limitless, but less so the options for charging them. Either we connect our mobile marvels to a cable, or we place them on a pad, where they couple to a magnetic near-field. David Smith and co-workers now argue that we might have a third option before too long: focused microwave beams.

The idea of beaming power from a source to a receiver is not new. The efficiency of the approach, however, is typically too low to be practically useful, because in the far field the aperture is limited by diffraction, making it difficult to target a given region.

To get around this problem, Smith et al. propose to work in the Fresnel zone, where a focus can be created. Their analysis shows that dynamically reconfigurable Fresnel lenses can be produced using flat metamaterials, known as metasurfaces. These could be conveniently mounted on walls or ceilings and should make it possible to focus energy onto several spots simultaneously. Also, current technologies based on liquid crystals or semiconductors might be used to build the required apertures at low cost.

\section{WHITE DWARFS}

\section{Pulsar in disguise}

Nat. Astron. 1, 0029 (2017)

In May 2015 a group of amateur astronomers spotted an unusual star shining brighter every 1.97 minutes in a wide spectrum from ultraviolet to radio wavelengths. AR Scorpii is a binary system made of a cold red dwarf and a fast-spinning white dwarf, orbiting each other every 3.55 hours. But why is it blinking?
Using polarization measurements from the South African Astronomical Observatory 1.9-m telescope, David Buckley and colleagues have explained the mechanism behind it.

Pulsars are fast-spinning neutron stars whose strong magnetic field accelerates charged particles that emit synchrotron radiation. The white dwarf in AR Scorpii has a strong magnetic field and rotates rapidly, so a similar thing happens. However, the system is more complex due to the additional magnetic interactions with the other star. The combined effect leads to the pulsed emission. Pulsars were first discovered fifty years ago and a similar behaviour was predicted for white dwarfs, but AR Scorpii is the first one to be spotted.

\section{COMPLEX NETWORKS}

\section{Hidden influence}

Nat. Commun. 8, 14103 (2017)

The old maxim that it's less about what you know than who you know is an appropriate analogy for how the behaviour of a complex network can be profoundly influenced by the weights of its links. Making friends is undoubtedly a different experience when the social network is populated by power brokers who club together - rather than individuals of equal standing. In the case of unweighted networks, the idea that they are embedded in hidden metric spaces has helped us to understand why certain links are connected. And now, Antoine Allard and co-workers have shown that this formalism can be extended to shed light on how these links are weighted.

Allard et al. looked at empirical data mapping a range of real biological, economic and transportation networks, and found evidence that the weight structure of these
TOPOLOGICAL SEMIMETALS

\section{Field-free quantum oscillations}

When strong magnetic fields are applied to metals, the electron states can become quantized, causing many thermodynamic and transport properties to exhibit periodic oscillations - the cyclotron orbits of the electrons become quantized into Landau levels. Tianyu Liu and colleagues predict that it should be possible to see such quantum oscillations in topological semimetals without applying any magnetic fields.

Perhaps the best-known class of topological semimetal are Dirac semimetals, which can be considered as three-dimensional analogues of graphene, featuring electron bands with linear dispersion close to a Dirac point. Liu etal. use theoretical methods to show that in thin films of the Dirac semimetal cadmium arsenide, elastic strain acts as a chiral gauge potential, which means that applying strain is analogous to applying a magnetic field.

Using their proposed geometry, they predict that pseudomagnetic fields as large as $15 \mathrm{~T}$ could be realized - more than enough to quantize the electron states. As this effect relies on the linearly dispersing electron bands, it should work for other Dirac (as well as Weyl) semimetals, providing a new tool for mapping the Fermi surface of topological semimetals. LF

networks emerges from an embedded geometry. They then constructed a general class of weighted network embedded in hidden metric spaces, which proved capable of reproducing key measures associated with their empirical data - and revealing the nature of the coupling between the metric space and the weight structure. AK

\section{VIRUS DISASSEMBLY}

\section{D phase transitions go viral}

Phys. Rev. Appl. 7, 014005 (2017)

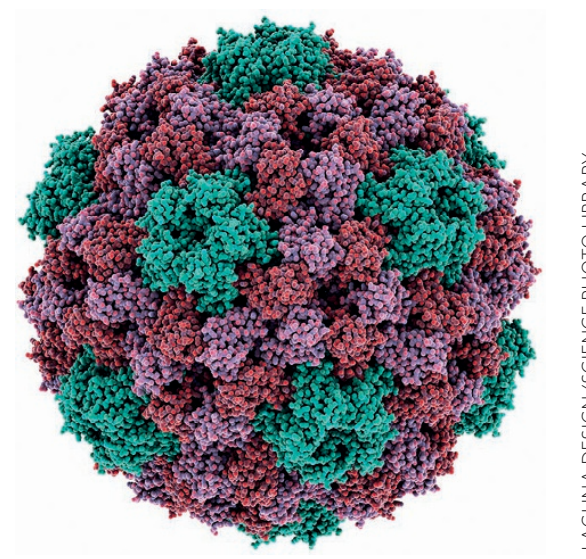

The genetic material of a virus is wrapped in a so-called capsid - a protective shell built from polypeptide chains. For half of the known viruses, the capsid's polypeptides are arranged into a structure with icosahedral symmetry, which is stabilized by the interplay between attractive and repulsive interactions in polypeptide subunits. Detailed knowledge of the parameters that govern these interactions enables an understanding not only of capsid formation, but also of their disassembly - important for devising strategies to prevent viral infection.

By mapping icosahedral capsid structures onto two-dimensional lattices, Guillaume Tresset and colleagues have found a way to describe the thermal dissociation of viral particles as a first-order phase transition. Monte Carlo simulations and a mean-field theory for capsid assembly led the authors to an analytical formula for the transition temperature as a function of the short-range attractive energy between subunits, their effective charge and the Debye screening length for the electrostatic interactions. Experiments with cowpea chlorotic mottle virus capsids (pictured), both empty and filled with RNA, corroborated the theoretical description providing a promising starting point for developing viral disassembly inhibitors.

Written by Luke Fleet, Iulia Georgescu, Abigail Klopper, Andreas H. Trabesinger and Bart Verberck. 